\title{
EXPERIENCE WITH A SIMPLE PLATE OVERLAP REDUCTION METHOD IN A FIELD AROUND ALPHA PERSEI
}

\author{
W. Dieckvoss \\ Hamgurger Sternwarte
}

Some years ago at Bergedorf we studied the field around Alpha Persei (Heckmann, O. et alt., 1956). In an area of $5^{\circ} \times 5^{\circ}$ more than 100 cluster members were found from an analysis of proper motions (p. m.) of 1453 stars. Meanwhile the field was studied photometrically (e.g. Mitchell, R.I., 1960 ). The age of the group seems to be comparable to that of the Pleiades (cp. e.g. Dachs, J., 1970), of the order of magnitude $10^{8}$ years.

Later on, with the AGK 3 in the making, we started work on a somewhat larger field - a circle of $10^{\circ}$ diameter - around Alpha Persei. New reduction of the relevant plates in the Astrographic Catalogue (A.C., "Carte du Ciel") and evaluation of new plates, both sets to be reduced by using AGK 3 data was begun. One should expect some results to show up as to the extension of the nucleus of the Perseus cluster.

The area in question is covered by 47 plates of the A.C. - zone Catania, and 16 plates from the A.C. - zone Helsing fors with epochs ranging from 1895.7 to 1905.1 . The plate centers are arranged in zones of $1^{\circ}$ distance in declination, in right ascension with intervals of $12 \mathrm{~m}$ north of and including $+49^{\circ}$, with intervals of $10^{\mathrm{m}}$ to the south. Thus with roughly $2^{\circ}$ distance in right ascension the sky is covered twice, as is the case in the whole A. C. with $2: 1 \times 2: 1$ plate areas.

At Bergedorf the rectangular coordinates $\mathrm{x}, \mathrm{y}$ from the $\mathrm{A}$. $\mathrm{C}$. were punched on cards together with the stars' numbers and their magnitudes or diameters. The coordinates were corrected on account of systematic errors depending on $\mathrm{x}, \mathrm{y}$, and magnitude: In the zone Catania preliminary formulae derived by A.Günther and H.Kox (1970 a) were used, in the Helsingfors zone formulae published by H.K. Eichhorn (1956) were used.

In all there are 17,481 stars in the area including a small region surrounding the field under study. Using preliminary plate constants derived by A. Günther and H. Kox (definitive values to be published by these authors, $1970 \mathrm{~b}$ ) the $\mathrm{x}, \mathrm{y}$ were transformed into right ascension and declination and simultaneously into tangential coordinates referred to the center of the field: $3^{\mathrm{h}} 21^{\mathrm{m}},+49^{\circ} 7^{\prime}(1950.0)$.

1365 stars of the AGK 3 were used for an iterative procedure of least squares' solution involving the 63 plates individually. In the first step - the only one I can report now as relating to the title of this paper - I applied the simple method of using the reference stars only for the successive iterations while smoothing the positions of these stars corresponding to the partial overlap of neighboring plate areas. This simple method had been tested at Bergedorf Observatory (Dieckvoss, W., de Vegt, C. 1967) with stars' positions created by a random number generator to cover artificial 
plate fields. The value of this numerical test may be seen in the fact, that in this case the "true" positions are known to an arbitrary degree of precision, and the method applied after adding normal dispersions to the true coordinates showed a considerable enhancement of precision of the plate constants, and an excellent representation of the true values of the coordinates. In a first classical plate solution the six contants $a, b, \ldots, f$ conforming to the formulae

$$
\text { (1) } \begin{aligned}
& a+b x+c y=x \\
& d+e x+f y=Y
\end{aligned}
$$

as equations of condition, were derived, where $\mathrm{X}, \mathrm{Y}$ are the tangential coordinates calculated from the reference stars' data. The mean error of the residuals $\mathrm{v}_{\mathrm{X}}, \mathrm{v}_{\mathrm{Y}}$ stems from a combination of the mean errors of $\mathrm{X}, \mathrm{Y}$, and of $x, y$. The order of magnitude of the AGK 3 data extrapolated to approximately 1900 by means of the revised AGK 2 data is $\pm 0 ! 36$. These solutions will be called the Oth iteration.

For each plate now the positions of the reference stars may be calculated by the formulae (1), and new values for the $\mathrm{X}, \mathrm{Y}$ will result, that when inserted into the equations of condition would bring the $\dot{v}_{x}, v_{y}$ to zero. If the positions of each star appearing on at least two plates arre combined by taking the arithmetic mean, and when new plate solutions are performed with the new $\mathrm{X}, \mathrm{Y}$, one may expect the residuals to become smaller.

On account of the considerable range in epochs of the A. C. - plates not the positions themselves but rather the correction to the $\mathrm{p}$. $\mathrm{m}$. was subjected to the smoothing procedure. Four iterations were performed in all. Table 1 gives straight mean values for the 0 th and the 4 th iteration.

\section{Table 1}

Root Mean Square Errors (mse) for two iterations

$\begin{array}{lcrc}\text { Number of reference stars } & \begin{array}{c}\text { Average } \\ \text { No/Plate }\end{array} & \text { Iteration No. . } 0 & 4 \\ \text { 1. found by computer..2737 } & 43.4 & \pm 0 ! 442 & \pm 0 ! 181 \\ \text { 2. actually used ......2498 } & 39.6 & \end{array}$

After that arithmetic means were calculated for those 5036 stars that appeared on two or more plates. Measuring lists were produced for 11 fields of $200 \mathrm{~mm} \times 200 \mathrm{~mm}$, maximum distance from center $110 \mathrm{~mm}$, the fields referring to 22 plates taken with the Bergedorf Big Schmidt of a scale value $86 ! ' 15 / \mathrm{mm}$. 10977 stars are to be measured, indicating the usual overlap with the range of 5036 program-stars. At last the coordinates of the old epoch and those of the new epoch are to be evaluated with overlap reduction methods that involve all stars, not the reference stars alone.

The coordinates from the Schmidt-plates are being measured with the 
Mann-comparator installed at Bergedorf with two screws of $260 / 240 \mathrm{~mm}$ usable length. A double-eyepiece, no projection is used. There is some deliberation to add a new device for an automatic centering of the stars' images by an on-line-computer.

The coordinates are punched automatically on cards to the nearest micron. The mean error for two settings is of the order of \pm 0.6 micron. Each of the plates of each field is measured in two positions differing in position angle by $180^{\circ}$. At the present time calibration of the comparator is not finished; we have preliminary values only. Nowhere the systematic correction seems to be larger than one micron, in most regions it is smaller.

A really good evaluation of stars' coordinates from plates, especially if several plates are to be combined, calls for thorough investigation of screw errors, and, still more important, of the effect of way errors. The last aim should be a reduction of coordinates to a truly orthogonal system with identical scale values for both axes.

Surely the Schmidt with bent plates is not well adapted to large field astrometry, our new 5 - lense - Zeiss - objective (dimension 230/2060 mm, photovisually corrected with a built-in filter) and new astrog raph will, however, not be delivered before the fall of 1971, and meanwhile I hope that with a conscientious application of overlap reduction methods some drawbacks of the astrometric Schmidt-performance might be overcome.

In any case the final calculations have to wait until the p.m. of all program-stars have been derived at least preliminarily from the comparison of new and old epochs reduced in the way sketched in the foregoing paragraphs. Then the coordinates of the A.C. plates can be reduced to a common epoch, e.g. 1900.0.

At the moment a reduction of the A. C. $-x, y$ to an orthogonal system, i. e. strictly Cartesian coordinates, is under consideration. In 1954 (Heckmann, O. et alt.) the Paris zone of the A.C., $+21^{\circ}$ to $+24^{\circ}$ was subjected to this treatment involving 4 constants for each plate, because in Paris the members of differential refraction were well applied.

For the zones Helsingfors and Catania, that enter our problem, the question is open, especially so, as in the course of deriving systematic corrections to the coordinates depending on $x, y$ and magnitude (Günther et alt. , 1970 a) the linear members were omitted in the final correction-formulae. As has been stressed repeatedly, in wholesale overlap plate reduction methods restriction of number of unknowns to physically justified ones is of special importance.

Formerly announced rates of expansion in the field around Alpha Persei as well as in the Pleiades cluster turned out to be entirely spurious, after some correction even to be false (Dieckvoss, W. 1959). With a precision of \pm 0 ! $003 /$ year mean error and perhaps 500 cluster members, however, an expansion age might show up; this surmise seems to be possible from a preliminary mean error of $10^{2}$ of a linear expansion coefficient (")"year) found from 137 cluster members out of $614 \mathrm{AG}$-stars with mean 
errors of $\pm 0 ! 008 / \mathrm{yr}$ in $\mathrm{p} . \mathrm{m}$. Higher precision and larger number perhaps yield 10-fold precision for the coefficient, and this might lead to a value just on the verge of detectability.

\section{References}

Dachs, J. 1970, Astron. and Astroph. 5, 312.

Dieckvoss, W. 1959, Mitt. Astron. Gesellschaft 1959, 68.

Dieckvoss, W., de Vegt, C. 1967, Astron. Nachr. 290, 125.

Eichhorn, H. K. 1957, Astron. J. 62, 142.

Günther, A. , Kox, H. 1970 a, Astron. and Astroph. 4, 156.

Günther, A. , Kox, H. 1970 b, in print Astron. and Astroph. ,

supplement series.

Heckmann, O. , Dieckvoss, W. , Kox, H. 1954, Astron. J. 59, 143.

Heckmann, O. , Dieckvoss, W. , Kox, H. 1956, Astron. Nachr. 283, 109.

Mitchell, R. I. 1960, Astroph. J. 132, 68.

\section{DISCUSSION}

Eichhorn: What is the difference between your method and what Herget proposes to do?

Dieckvoss: I do not include the field stars in this first solution. I forgot something, I had in my paper. If you use the alternate method it is almost imperative not to use too many unknowns. And you saw, perhaps this morning, in the tables that give the plate constants for the astrographic catalogue from $40^{\circ}$ up to $90^{\circ}$ there are given the tangential point exactly defined by the center of the plate and then two values of the scale value, and two values of the orientation. And now it turned out, in the last few weeks or so, that when Gunther and Kox approximate the solution of the systematic errors of the different zones, they omitted in the final tables the linear members which were included in the representation of the residuals by polynomials in coordinates and diameters of the reference stars. And if you think about it, the linear members and the members of the third order are intercorrelated because once the coefficient is one times e.g. $\mathrm{x}$ or else $\mathrm{x}^{2}$ times $\mathrm{x}$. It is very much intercorrelated. And now it depends just on chance. You omit the linear member, you get members of the third order, and they are a bit wrong. Only together they give a good presentation of the mass of residuals. But you omit the coefficients of linear members that are taken care of in the solutions of the individual plates. But the old original measured coordinates with a good definition by reseau, refraction and similar systematic corrections may be considered as strictly orthogonal. Then you can be sure that you have to have only two unknowns - scale error and orientation of the plate - besides the additive constant that means the tangential point. And then we can have only four constants. It is a good thing to carry out your overlap solution. And we try to do this ourselves because we have all necessary data on magnetic tape. 
Eichhorn: Because what you have done here seems to me to be exactly well, except for taking the field star - this is almost the same thing that I proposed in December of 1960.

Dieckvoss: It is a very simple thing. It is a good idea...

Eichhorn: What you say seems to support the idea, but the convergence of the iterative procedure is very substantially improved if the ratio of the rates of the reference stars is larger than that of the field stars. Especially if it can be followed through by an output of the reference stars and of all the weights. And then a convergence. I am somewhat surprised that you get the improvement that you do; this is probably due to the simplicity of the mode. You are arguing with numbers.

Herget: Wait a minute, I think there is. From each successive iteration you throw out the ones that are bigger than three sigma. The improvement from 0!'442 to 0!'181 in the successive approximations is due in part to the largest residuals being eliminated by the three-sigma-test.

Dieckvoss: The first iteration helps; the others, second to the fourth, do not bring much.

Herget: But the value you had for the RMS error, if you had taken out the erroneous data which the three-sigma-test threw out you would get what amounts to the value you get in the fourth iteration.

Dieckvoss: You see there are many other things involved. The three-sigmatest is applied in each iteration separately, all data enter the solutions, and in most cases just gross errors are eliminated. Diminishing the sigma itself occurs because the reference stars' positions are adapted to the plate data.

Herget: In other words, I think there is no guarantee that we know how to interpret the improvement of that number.

Dieckvoss: Yes, to omit those large residuals is only a standard procedure.

Herget: I know it, but suppose that these really are errors in the data, poor measurement, or something. Suppose you had not used them in the first place. Then the zero approximation would have come out.

Dieckvoss: Yes.

Herget: So I think it should not be just concluded unilaterally that there is an improvement because of the method. There may be, but there also may not be. 
Dieckvoss: Yes, but we have studied all those 63 plates. There are some differences, ones that make three solutions, four, and so on.

Murray: How many members of the Alpha-Persei association are there in this field? Could you use those alone as your reference system?

Dieckvoss: I hope so. But I will attempt to do it because I will perhaps find an expansion age around $10^{8}$ years, but I do not really think I can find it.

Roman: To ask a somewhat physical question. First, do you find any variation in motion from me mber to member other than that attributable easily to the errors of the proper motion? And, secondly, what are the colors of the faint stars you have observed?

Dieckvoss: We are just beginning; we still have to measure the new plates to find the answers to those questions. 